\title{
Utilização de medicamentos e plantas medicinais por gestantes atendidas na unidade de saúde da mulher em Alegre, ES, Brasil
}

\author{
Use of drugs and medicinal plants by pregnant women assisted in \\ the women's health unit in the city of Alegre, ES, Brazil
}

Recebido em: 24/04/2017

Aceito em: 29/08/2017

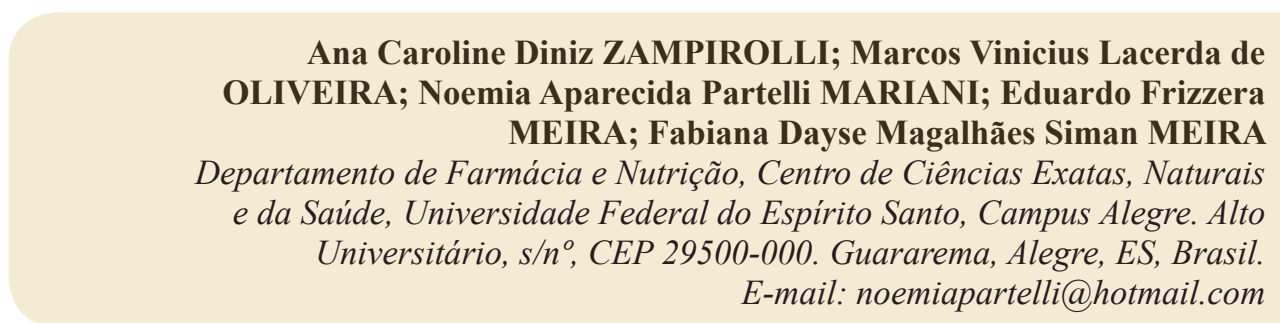

ABSTRACT

Self-medication still is a common practice in Brazil. Due to the risks that this practice can cause in pregnant women, a study was carried out to identify the consumption of drugs and medicinal plants by pregnant women assisted in the Women's Health Unit, in the city of Alegre, ES. The study was conducted through interviews. A total of 115 pregnant women aged between 14 and 43 years were interviewed. Of these, $99.1 \%$ were literate and $48.69 \%$ of pregnant women did not perform paid activity. Most of the women were in the third trimester of pregnancy. Of the interviewed women, $80 \%$ said they had used at least one medication during pregnancy, only $64 \%$ of women said they knew the purpose of the prescribed medicine, and $21 \%$ were by self-medication. It was found that $9.5 \%$ had some type of chronic disease. The most commonly used drugs were antianemics and non-opioid analgesics. According to FDA risk classification, $13.2 \%$ of pregnant women used medications that may cause adverse effects. With regard to medicinal herbs consumption, $17 \%$ used at least one type during the gestational period, and the most used was lemon-grass. It was possible to note that self-medication is part of the routine of pregnant women in the city of Alegre, evidencing the need for pharmaceutical guidance.

Keywords: Self Medication; Pregnant Women; Medicinal teas; Risk Groups.

\section{RESUMO}

A automedicação é um problema universal, portanto, gestantes também estão sujeitas a utilizarem medicamentos constantemente. Assim, o objetivo desse trabalho foi identificar a utilização de medicamentos e plantas medicinais junto às gestantes atendidas na Unidade Saúde da Mulher, no município de Alegre, ES. O estudo foi realizado por meio de entrevistas. Foram entrevistadas 115 gestantes, e a idade variou de 14 a 43 anos. Dessas, 99,1\% eram alfabetizadas, e 48,69\% não realizavam atividade remunerada. A maioria das gestantes tinha renda familiar baixa. Em relação ao tratamento de água e coleta de lixo, $24 \%$ relataram utilizar água de poços e $18 \%$ não contavam com a coleta de lixo semanal. A maior parte das gestantes estava no terceiro trimestre da gravidez. Das entrevistadas, $80 \%$ afirmaram utilizar pelo menos um medicamento na gestação, sendo que somente $64 \%$ das gestantes afirmaram saber a finalidade do medicamento prescrito e $21 \%$ utilizaram por automedicação. Foi constatado que $9,5 \%$ apresentavam algum tipo de doença crônica. Os medicamentos mais utilizados foram antianêmicos e analgésicos não opióides. De acordo com a classificação de risco do FDA, 13,2\% das gestantes utilizavam medicamentos que podem causar efeitos adversos. No que se refere ao consumo de chás, $17 \%$ afirmaram utilizar no mínimo um tipo durante o período gestacional, e o mais utilizado foi o de capim-cidreira. Foi possível notar que a automedicação faz parte do cotidiano das gestantes no município de Alegre, evidenciando a necessidade da orientação farmacêutica.

Palavras-chave: automedicação; gestantes; chás medicinais; grupos de riscos 


\section{INTRODUÇÃO}

A automedicação é uma prática comum no Brasil, sendo amplamente discutida no âmbito médico-farmacêutico, tendo ganhado destaque na área da saúde (1). Devido a sua prevalência, os pacientes criam independência e autonomia para lidar com o tratamento de doenças menos graves, contando ou não com a orientação médica ou farmacêutica $(1,2)$.

Como a automedicação é um problema universal, as gestantes também estão sujeitas a constantemente utilizar medicamentos e esta exposição não envolve somente o corpo materno, mas também o corpo do feto. A resposta farmacológica após a administração do medicamento é distinta ao se relacionar o corpo materno do corpo fetal, portanto, os cuidados devem ser criteriosos para que não ocorram danos no desenvolvimento do embrião (3-5). A barreira placentária não é eficaz para conter a maioria dos fármacos, e uma vez atravessando-a, os efeitos decorrentes dependem da capacidade, do feto, de metabolizar o fármaco e da habilidade dos órgãos em não sofrer a agressão; por esses motivos, a automedicação durante o período gestacional deve ser evitada (6).

Apesar de apresentarem um elevado risco para as gestantes, os medicamentos são comumente utilizados. Durante o período gestacional é possível observar o surgimento de manifestações clínicas inerentes à própria gravidez, além de doenças crônicas ou intercorrentes, como hipertensão e diabetes, e a própria automedicação. Dessa forma, esses fatores podem explicar a constante busca por medicamentos $(5,7)$. Diante de todos os riscos envolvidos na utilização de medicamentos, muitas gestantes recorrem ao uso de plantas medicinais, acreditando que estas não causam mal ao feto. Todavia, há uma escassez de pesquisas que envolvam a utilização destes produtos durante a gravidez, e assim, também são escassas as informações quanto à segurança no uso. Por isso, a utilização de plantas medicinais deve ser feita de forma racional e orientada por profissional especializado (8).

Existe ainda a crença popular que o "natural" é inofensivo à saúde; no entanto, a formulação química de um produto de origem vegetal é complexa e, assim, muitos constituintes não são identificados. Dessa forma, os riscos envolvidos na sua utilização são elevados pelo fato dos efeitos no organismo serem, em certos casos, desconhecidos (8).

Considerando que entrevistas populacionais são meios significativos para a obtenção de dados em relação à utilização de medicamentos e plantas medicinais por gestantes, e a urgência na elaboração de métodos que esclareçam seus riscos, principalmente quando é realizada a automedicação, o presente trabalho teve a finalidade de identificar o padrão de consumo de medicamentos e plantas medicinais das gestantes atendidas na Unidade Saúde da Mulher, localizado no município de Alegre, ES.

\section{MÉTODOS}

O estudo realizado foi do tipo transversal quantitativo, analisando a terapêutica das gestantes atendidas na Unidade de Saúde da Mulher, localizada no município de Alegre, ES, por meio de um questionário. O trabalho foi baseado em um estudo observacional, não havendo intervenção.

Foram entrevistadas 115 gestantes, que estavam cadastradas na Unidade, no período de setembro a dezembro de 2014. A coleta de dados foi realizada por meio de um questionário, aplicado às gestantes antes das consultas ambulatoriais de pré-natal para se obter as seguintes variáveis: dados pessoais, como data de nascimento e escolaridade; dados socioeconômicos, como situação conjugal, profissão, número de filhos, renda familiar, coleta de lixo e água tratada; dados sobre a gestação, incluindo o uso de medicamentos e chás, semanas de gestação, medicamentos prescritos e os utilizados pela prática da automedicação; sintomas da gravidez; dados sobre o hábito de vida, analisando o uso de substâncias ilícitas e consumo de bebida alcoólica ou tabaco. Após a realização do questionário, as gestantes receberam um panfleto informativo indicando os riscos da automedicação durante o período gestacional.

O trabalho foi aprovado pelo Comitê de Ética da Universidade Federal do Espírito Santo sob o número 33836214.5.0000.5060. As participantes da pesquisa foram esclarecidas quanto aos objetivos do questionário e assinaram o Termo de Consentimento Livre e Esclarecido (TCLE), após o aceite voluntário em participar do trabalho. Os resultados foram analisados com auxílio do programa Microsoft Excel.

\section{RESULTADOS E DISCUSSÃO}

A idade entre as gestantes entrevistadas variou de 14 a 43 anos, com uma média de 25,1 anos. Entre as participantes, $21,7 \%$ corresponderam às gestantes com idade menor ou igual a 20 anos. Dentre estas, $8,7 \%$ ti- 
nham entre 14 e 17 anos, representando as gestantes menores de idade. As gestantes com idade acima de 30 anos corresponderam a $22,6 \%$ e aquelas que possuíam idade superior a 35 anos representaram $6,9 \%$.

No quesito escolaridade, a grande maioria mostrou ser alfabetizada, totalizando $99,1 \%$; apenas $0,8 \%$ afirmou ser analfabeta. Dentre as alfabetizadas, $37,4 \%$ das gestantes possuíam ensino médio completo. No entanto, este valor foi semelhante ao nível de ensino fundamental incompleto, que correspondeu a 31,3\%. Vale destacar que apenas 10,4\% destas apresentavam mais de 11 anos de estudos. Em relação à ocupação das entrevistadas, foi verificado que $48,7 \%$ das gestantes não realizavam atividade remunerada, desempenhando somente atividade do próprio lar; e $45,2 \%$ realizavam atividade remunerada, em diferentes funções, como enfermeira, balconista, professora, operadora de telemarketing e autônoma.
Sobre seus respectivos salários, 48,7\% recebiam até um salário mínimo mensal e 48,6\% recebiam entre um a sete salários, sendo que dessas $41,7 \%$ recebiam de um a três salários mínimos. Assim, os salários mensais variavam de $\mathrm{R} \$ 724,00$ a $\mathrm{R} \$ 2.172,00$. A maioria das gestantes pertencia a uma situação financeira relativamente baixa, constatando que $19,1 \%$ possuíam de dois a quatro filhos e que $0,8 \%$ possuíam sete filhos anteriores àquela gestação. A renda familiar das entrevistadas foi baseada em um salário mínimo de R \$724,00.

As gestantes entrevistadas relataram as condições de moradia, incluindo informações sobre a presença de tratamento de água e coleta de lixo em suas residências. De acordo com o relatado, $76 \%$ das gestantes afirmaram receber água tratada e $82 \%$ afirmaram contar, semanalmente, com o serviço de coleta de lixo. Porém, 24\% relataram não possuir água potável em casa e sim, água proveniente de poços, e 18\% não contavam com a coleta semanal de lixo.

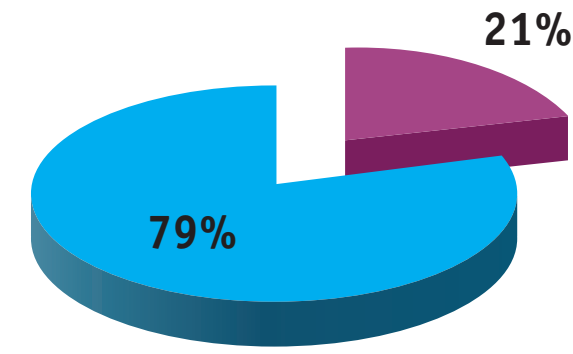

Pratica a automedicação (19)

Não pratica a automedicação (73)

Figura 1. Distribuição das gestantes atendidas na Unidade Saúde da Mulher quanto à utilização de medicamentos durante a gravidez, em Alegre, ES (2014)

De acordo com os dados coletados, $19,1 \%$ das gestantes encontravam-se no primeiro trimestre de gravidez, 35,7\% estavam no segundo trimestre e 44,3\% apresentaram-se no terceiro trimestre. Apenas uma gestante não soube responder em que período se encontrava, representando $0,8 \%$. O presente estudo mostra que parte das gestantes iniciou o pré-natal antes mesmo de completar o quarto mês de gravidez, destacando o início precoce da assistência.

Das 115 gestantes entrevistadas, $80 \%$ afirmaram utilizar pelo menos um medicamento durante o período gestacional e 19\% relataram não utilizar.
Isso é justificável pelo fato da assistência pré-natal nas unidades de saúde facilitar o acesso aos medicamentos, uma vez que a distribuição ocorre de forma gratuita nas farmácias básicas. Nesse contexto, 21\% das gestantes que relataram utilizar medicamentos praticavam a automedicação e $79 \%$ utilizavam medicamentos apenas sob prescrição médica (Figura 1). Ao ser pesquisado sobre o conhecimento a respeito da indicação, 64\% das gestantes afirmaram saber a finalidade do medicamento prescrito e $36 \%$ não tinham conhecimento sobre o motivo da indicação clínica. 
Tabela 1. Distribuição dos medicamentos utilizados pelas gestantes atendidas na Unidade Saúde da Mulher de acordo com sua classe farmacológica, em Alegre, ES (2014)

\begin{tabular}{|l|l|r|}
\hline Classe Farmacológica & \multicolumn{1}{|c|}{ Medicamento } & Frequência (\%) \\
\hline Analgésicos não opióides & paracetamol, dipirona & 16,3 \\
\hline Antiácidos & hidróxido de alumínio & 0,4 \\
\hline Antianêmicos & ácido fólico, sulfato ferroso, glicinato férrico & 56,1 \\
\hline Antibacterianos & nitrofurantoína, espiramicina & 0,9 \\
\hline Anticoagulantes & enoxaparina & 0,4 \\
\hline Antidepressivos & Clonazepam, dimenidrinato & 0,4 \\
\hline Antieméticos & bromoprida, cloridrato de meclizina & 2,3 \\
\hline Antiespasmódicos & escopolamina & 0,4 \\
\hline Antiflatulentos & dimeticona & 0,4 \\
\hline Anti-hipertensivos & metildopa, hidralazina & 2,8 \\
\hline Anti-inflamatório & diclofenaco & 0,4 \\
\hline Hormônio Tireoidiano & levotiroxina & 0,4 \\
\hline
\end{tabular}

Do total de gestantes entrevistadas, 9,5\% possuía algum tipo de doença crônica anterior à gestação. As doenças citadas foram: hipertensão arterial sistêmica $(5,2 \%)$, depressão $(0,9 \%)$, hipotireoidismo $(0,9 \%)$, trombose $(0,9 \%)$ e toxoplasmose $(0,9 \%)$. Vale ressaltar que uma gestante $(0,9 \%)$ relatou esquizofrenia, no entanto, a mesma não soube responder qual medicamento utilizava.

A Tabela 1 mostra a relação dos medicamentos mais utilizados sob prescrição médica, com a sua classificação farmacológica. Dentre os grupos farmacológicos mais utilizados, destacaram-se os antianêmicos (ácido fólico e sulfato ferroso), com $56,1 \%$, seguidos dos analgésicos não opióides (paracetamol e dipirona), com $16,3 \%$. Os antieméticos e os anti-hipertensivos representaram $2,3 \%$ e $2,8 \%$, respectivamente.

$\mathrm{O}$ presente estudo mostra que o fármaco mais utilizado pela prática de automedicação foi o paracetamol, com $54,5 \%$ do total usado sem prescrição médica. Logo em seguida encontra-se a dipirona com $31,8 \%$, a escopolamina com $9 \%$ e o diclofenaco com $4,5 \%$.

De acordo com Guerra e cols.(2008), a utilização de medicamentos no período gestacional, é um desafio contínuo para a classe médica/farmacêutica, dado que os riscos existentes não estão relacionados somente à mulher, mas também ao concepto (9). Nesse mesmo trabalho, foi mostrado que $86,6 \%$ das mulheres entrevistadas utilizaram pelo menos um medicamento durante a gravidez.

Com o intuito de conduzir os profissionais prescritores na escolha farmacêutica mais indicada para uma gestante, a agência americana Food and Drug Administration (FDA) classificou os fármacos de acordo com seus efeitos na gestação em categorias de risco A, B, C, D e X10. O Quadro 1 mostra a classificação dos medicamentos prescritos ou não, utilizados pelas gestantes, segundo a classificação do FDA.

Quadro 1 Fármacos utilizados pelas gestantes de Alegre, ES (2014) e a classificação de risco de acordo com a Food and Drug Admistration.

\begin{tabular}{|l|l|l|l|l|}
\hline \multicolumn{1}{|c|}{ Risco A } & \multicolumn{1}{|c|}{ Risco B } & \multicolumn{1}{c|}{ Risco C } & Risco D \\
\hline & paracetamol & & \\
\hline & dipirona & & \\
\hline $\begin{array}{l}\text { ácido fólico } \\
\text { sulfato ferrosol }\end{array}$ & dimenidrinato & escopolamina & \\
\hline levotiroxina & nitrofurantoína & hidralazina & \\
\hline dimeticona & metildopa & cloridrato de meclizina & clonazepam \\
\hline & hidróxido de alumínio & bromoprida & \\
\hline & enoxaparina & vitamina c & \\
\hline & dimenidrinato & & \\
\hline
\end{tabular}


Os medicamentos mais prescritos compreenderam as classes $\mathrm{A}$ e $\mathrm{B}$, que não apresentam efeitos adversos às gestantes. Dentre estes se destacaram os antianêmicos, que são medicamentos adotados pelo Ministério da Saúde para assistência pré-natal (11). Segundo a Organização Mundial de Saúde (OMS), deve-se administrar medicamentos a base de ferro a mulheres nos últimos quatro a cinco meses de gestação, justificando assim a alta incidência de prescrição do sulfato ferroso no presente estudo (1).

Considerando os medicamentos prejudiciais a saúde fetal, 13,2\% das gestantes utilizaram medicamentos que podem causar efeitos adversos relevantes, sendo $12,7 \%$ pertencentes à classe de risco $\mathrm{C}$ (escopolamina, hidralazina, cloridrato de meclizina, bromoprida e vitamina $\mathrm{C}$ ), e $0,5 \%$ às classes de risco $\mathrm{D}$ (clonazepam). Nenhum medicamento foi classificado como risco potencial de teratogenicidade, que são os fármacos que se enquadram dentro da classe $\mathrm{X}$.

Não foram observados efeitos adversos graves decorrentes do uso da hidralazina na gravidez humana. Porém, experimentos têm mostrado um potencial teratogênico em camundongos (12). Estudos epidemiológicos também não observaram aumento no risco de anormalidades fetais com o uso da meclizina. Entretanto, estudos em ratos mostraram que a meclizina causa fenda palatina quando administrada em uma dose correspondente a $25-50$ vezes a dose recomendada em humanos (13).
Em relação à bromoprida, é secretada pelo leite materno, e assim, sua utilização em grávidas e lactentes não é recomendada, mesmo sem evidências concretas de ris$\cos (14)$. Quanto ao clonazepam, foi verificado um caso de cianose persistente nos dez primeiros dias pós-parto em uma criança que foi exposta intra-útero e por meio da amamentação (15).

Em relação aos medicamentos utilizados sem prescrição, os mais frequentes foram das classes $\mathrm{B}$ e C, como o paracetamol e dipirona, escopolamina e diclofenaco, respectivamente. Apesar da dipirona se encontrar na classe de risco B, é um fármaco que deve ser evitado no período gestacional, principalmente no último trimestre de gravidez, uma vez que é capaz de fechar prematuramente o ducto arterial e causar complicações perinatais por alterar a agregação plaquetária da mãe e do recém-nascido (16).

Sobre o consumo de medicamentos durante a gestação, é possível notar que nenhum medicamento é isento de toxicidade. Dessa forma, o acompanhamento dos medicamentos após a sua introdução no mercado é imprescindível, entrando em cena a farmacovigilância $(17,18)$.

Além da prescrição médica e farmacovigilância, é necessária a presença do farmacêutico no recinto profissional, garantindo a eficácia e segurança durante a utilização do medicamento (19). Além disso, a presença do profissional farmacêutico resgata a verdadeira assistência farmacêutica, promovendo a interação direta com o paciente, e uma farmacoterapia racional, reforçando o seu valor como profissional de saúde20.
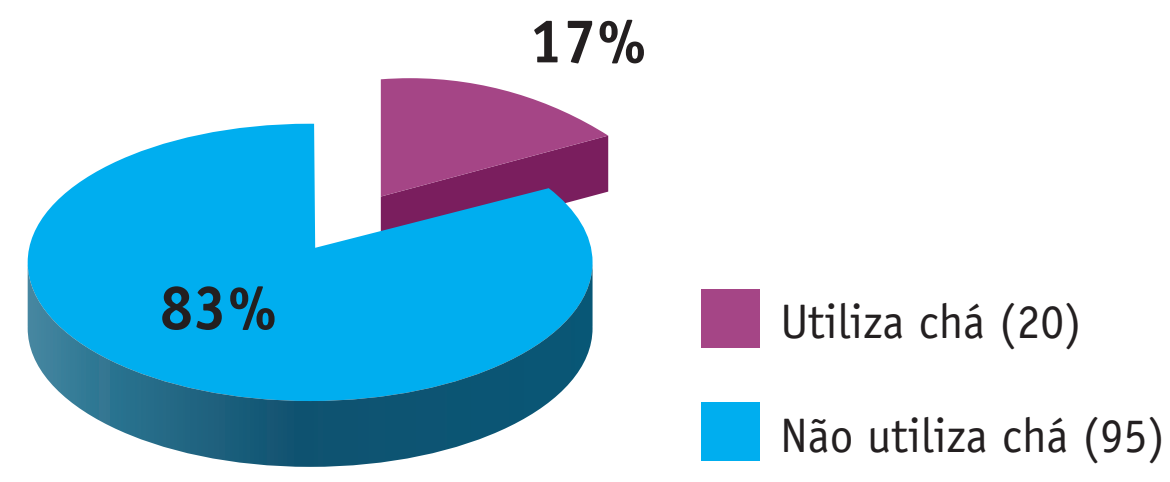

Figura 2. Distribuição das gestantes atendidas na Unidade Saúde da Mulher em relação ao uso de chás, em Alegre, ES (2014) 
Tabela 4. Distribuição dos chás utilizados pelas gestantes atendidas na Unidade Saúde da Mulher, Alegre-ES, 2014.

\begin{tabular}{|l|l|l|l|}
\hline \multicolumn{1}{|c|}{ chá } & \multicolumn{1}{c|}{ nome cientifico } & gestantes & \multicolumn{1}{c}{ ações } \\
\hline capim cidreira & Cymbopogon citrates (D.C.) Stapf & 11 & Relaxante da musculatura uterina \\
\hline erva doce & Pimpinella anisum L. & 10 & Antiespasmódico \\
\hline hortelã & Mentha piperita L. & 5 & Abortiva; digestiva, carminative \\
\hline camomila & Matricaria recutita L. & 3 & Antiespasmódico, anti-inflamatório, tópico, distúrbios digestivos \\
\hline poejo & Mentha pulegium L. & 1 & Óleo altamente tóxico, abortivo, vermífugo \\
\hline alecrim & Rosmarinus officinalis L. & 1 & Características emenagogas, cicatrizantes e abortivas \\
\hline
\end{tabular}

No que se refere ao consumo de chás, $17 \%$ das mulheres utilizavam pelo menos um tipo durante o período gestacional, como mostra a Figura 2. E a Tabela 3 relaciona todos os chás citados com seu potencial risco quando utilizado na gestação.

$\mathrm{Na}$ distribuição das gestantes em relação ao conhecimento sobre as propriedades dos chás, $85 \%$ afirmaram saber sobre seus respectivos efeitos e $15 \%$ utilizavam sem ter esse conhecimento. Além disso, foi verificado que $36 \%$ de todas as gestantes que fizeram uso de pelo menos um chá ou medicamento durante a gestação, nunca foram alertadas sobre os inerentes riscos envolvidos.

$\mathrm{O}$ efeito esperado mais citado pelas gestantes ao utilizarem plantas medicinais foi o calmante, pois muitas relataram estar agitadas e ansiosas. A planta mais utilizada foi Cymbopogon sp. (capim cidreira); todavia, além do efeito calmante, o capim cidreira possui como efeito adverso a ação abortiva por possuir a propriedade de relaxamento do útero $(20,21)$.

Dentre as plantas citadas pelas gestantes, a Pimpinella anisum L. (erva-doce) foi a segunda. Analisando os mecanismos de interação medicamento versus planta medicinal, a erva-doce é capaz de causar sedação leve quando utilizada na forma de chá, e associada a fármacos hipnóticos pode prolongar a ação do medicamento (22).

A Matricaria recutita L. (camomila) pode interagir com anticoagulantes e barbitúricos. Essa espécie é capaz de aumentar os riscos de sangramento quando utilizada com anticoagulantes, como a varfarina; e quando utilizada com barbitúricos, é capaz de atuar em sinergismo com o fármaco, prolongando o efeito depressor do sistema nervoso central (22).

A toxicidade oriunda da utilização de plantas pode trazer sérios riscos à saúde. Apesar de parecerem simples quando comparados aos tratamentos convencionais, os efeitos adversos podem ser problemas de saúde pública (19). Algumas espécies vegetais são potencialmente perigosas quando utilizadas durante a gravidez. Existem estudos onde espécies que apresentam antraquinonas em sua composição possuem um risco potencial elevado. As antraquinonas (de sene, cáscara e frângula) são capazes de induzir contrações uterinas, aumentando o fluxo sanguíneo para o útero e seus anexos, aumentando assim as chances de perda do feto (8).

Além disso, os constituintes presentes nas plantas medicinais são capazes de interagir com outros fármacos ou alimentos, trazendo assim diferentes efeitos adversos. As características individuais, tais como idade, sexo, condições fisiológicas e as características genéticas também são capazes de alterar o real efeito do produto natural (22). Além do mais, o consumo desses produtos pode alterar a eficácia de tratamentos convencionais, potencializando ou inibindo a ação esperada de um medicamento (22).

Em relação aos hábitos de vida das gestantes entrevistadas, foi constatado que $6,9 \%$ eram tabagistas e consumiam bebida alcoólica pelo menos uma vez por semana; $6,9 \%$ relataram ser fumantes ativas e $0,8 \%$ afirmou fumar pelo menos uma vez ao mês. Se tratando do consumo de bebida alcoólica, 4,3\% das gestantes afirmaram consumir álcool pelo menos aos fins de semana, $1,7 \%$ consumiam pelo menos uma vez ao mês e $0,8 \%$ consumia bebida alcoólica todos os dias. Dessa forma, $1,7 \%$ das gestantes consumiam bebida alcoólica e fumavam.

Sabe-se que o efeito do álcool em recém-nascidos se manifesta a partir da Síndrome Fetal Alcoólica, que acomete até $33 \%$ das crianças oriundas de mães que fizeram uso de até 150 gramas de etanol por dia. Quando ingerido de forma abusiva nas primeiras semanas de gestação, pode estar envolvido em abortos espontâneos, e o seu consumo no decorrer dos meses pode causar má formação física, implicando também na má formação do tubo neural $(23,24)$.

Vários estudos têm mostrado a alta prevalência do consumo de bebida alcoólica e fumo por gestantes. Um estudo em diferentes capitais brasileiras mostrou 
que $34,4 \%$ das gestantes consumiam bebida alcoólica e $17,5 \%$ eram fumantes (25). Esse estudo ainda mostrou a associação entre o fumo na gestação e algumas variáveis como idade, escolaridade, paridade, consumo de bebidas alcoólicas, situação conjugal e geográfica (25). Moraes e Reichenheim (2007) mostraram que $40,6 \%$ das mulheres entrevistadas fizeram uso de bebida alcoólica durante algum período da gestação, enquanto que 10,1\% relataram ter consumido álcool até o final da gestação. A alta prevalência do uso de álcool na gestação indica importante problema de saúde pública e deve ser rastreada durante a assistência pré-natal (26).

Ao ser questionada a prática de exercícios físicos, somente $30,4 \%$ das gestantes relataram que frequentemente caminhavam durante o dia, seja por lazer ou por trabalho. Daquelas que praticavam alguma atividade, $20 \%$ afirmaram realizar todos os dias, com tempo variado de 20 a 40 minutos de caminhada, e 10,4\% praticavam a caminhada pelo menos de duas a três vezes na semana, no período de 30 a 40 minutos. A atividade física regular durante a gravidez melhora ou mantém a aptidão física, auxilia no controle de peso além de reduzir o risco de diabetes gestacional em mulheres obesas e melhorar o bem-estar psicológico (27). Ao verificar os principais sintomas da gravidez relatados, a falta de ar, clinicamente denominada dispneia, esteve presente em relatos de gestantes com mais de 24 semanas, enquanto náuseas, vômitos e tontura, se manifestaram em gestantes entre a quarta e a décima segunda semana de gestação.

Diante de todo o exposto, é fato que o consumo irracional de medicamentos e plantas medicinais é questão de saúde pública, principalmente no que se refere ao uso por gestantes. Os estudos epidemiológicos evidenciam a necessidade da formulação de programas educa- tivos e políticas públicas pelos profissionais de saúde, ou seja, intervenções que possam minimizar o consumo exacerbado de medicamentos durante o período gestacional (19).

\section{CONCLUSÃO}

O presente estudo mostrou que as gestantes necessitam de uma orientação especial dos profissionais de saúde, tendo em vista que tanto os medicamentos quanto as plantas medicinais utilizadas de forma irracional, podem gerar graves danos à saúde da gestante e do feto.

Além disso, o estudo da utilização de medicamentos em uma população de risco, como as gestantes, é de fundamental importância, pelo fato de viabilizar intervenções educativas dirigidas a essa população e atividades de orientação continuada para profissionais de saúde, promovendo a conscientização da sociedade e da equipe de saúde sobre a importância do uso racional de medicamentos.

Dessa forma, é fundamental a inserção de um profissional farmacêutico na atenção primária à saúde, de modo a garantir o fornecimento de informações essenciais no ato da dispensação.

\section{AGRADECIMENTOS}

Às gestantes da Unidade de Saúde da Mulher de Alegre, ES, que aceitaram participar do presente estudo, à secretária de Saúde Florinette Pinto Ridolphi, à Secretaria Municipal de Saúde de Alegre e à Universidade Federal do Espírito Santo.

\section{REFERÊNCIAS}

1. WHO. World Health Organization. The benefits and risks of self-medication. WHO Drug Information 2000; 14(1): $1-76$.

2. Hughes CM, McElnay JC, Fleming GF. Benefits and risks of self-medication. Drug Saf. 2001; 24(14): 10271037. DOI: 10.12691/ajps-2-1-4.

3. Gomes KR, Moron AF, Silva RS, Siqueira AA. Prevalência do uso de medicamentos na gravidez e relações com as características maternas. Rev. Saúde Pública. 1999; 33(3): 246-254. DOI: 10.1590/S003489101999000300005 .
4. Maeda ST, Secoli SR. Utilização e custo de medicamentos em gestantes de baixo-risco. Revista Latino-Am. Enfermagem. 2008; 16(2): 266-271. DOI: 10.1590/S010411692008000200015 .

5. Dawes M, Chowienczyk PJ. Pharmacokinetics in pregnancy. Best Practice \& Research Clinical Obstetrics and Gynaecology. 2001; 15(6): 819-826. DOI: 10.1053/ beog.2001.0231.

6. Osorio De Castro CGS, Pepe VLE, Luiza VL, Cosendey MAE, Freitas AM, Miranda FF, Bermudez JAZ, Leal MC. Uso indicado e uso referido de medicamentos du- 
rante a gravidez. Cad. Saúde Pública. 2004; 20(1): 573582. DOI: $10.1590 / \mathrm{S} 0102-311 \mathrm{X} 2004000700008$.

7. Clarke JHR, Rates SMK, Bridi R. Um alerta sobre o uso de produtos de origem vegetal na gravidez. Infarma. 2007; 19(1,2): 41-48.

8. Rates SMK. Metilxantinas. In: Simoes CMO, Schekel LP, Gosmann G, Mello JCP. Farmacognosia da planta ao medicamento. 5 ed. Florianópolis: UFRGS; 2003. p.885-901.

9. Guerra GCB, Silva AQB, França LB, Assunção PMC, Cabral RX, Ferreira AAA. Utilização de medicamentos durante a gravidez na cidade de Natal, Rio Grande do Norte, Brasil. Rev. Bras. Ginecol. Obstet. 2008; 30(1): 12-18. DOI: 10.1590/S0100-72032008000100003.

10. Rodrigues AVP, Terrengui LCS. Uso de medicamentos durante a gravidez. Rev. UNISA. 2006; 7: 9-14.

11. BRASIL. Ministério da Saúde. Assistência pré-natal: manual técnico. Brasília: Ministério da Saúde; 2000.

12. BRASIL. Agencia Nacional de Vigilância Sanitária (ANVISA), 20 de fevereiro de 2015. Bulário Eletrônico - Cloridrato de Hidralazina. Laboratório Novartis. MS -1.0068 .0013 .

13. BRASIL. Agencia Nacional de Vigilância Sanitária (ANVISA), 20 de fevereiro de 2015. Bulário Eletrônico - Meclizina. Laboratório Apsen Farmacêutica. MS -1.0118 .0165 .

14. BRASIL. Agencia Nacional de Vigilância Sanitária (ANVISA), 20 de fevereiro de 2015. Bulário Eletrônico Bromoprida. Laboratório NovaFarma. MS -1.1402.0067.

15. BRASIL. Agencia Nacional de Vigilância Sanitária (ANVISA), 20 de fevereiro de 2015. Bulário Eletrônico - Clonazepam. Laboratório NovaFarma. MS -1.5651.0029.

16. BRASIL. Agencia Nacional de Vigilância Sanitária (ANVISA), 20 de fevereiro de 2015. Bulário Eletrônico-dipirona sódica. Laboratório PratiDonaduzzi. MS 0156688/15-1.

17. Rocha RS, Bezerra SC, Lima JWO, Costa FS. Consumo de medicamentos, álcool e fumo na gestação e avaliação dos riscos teratogênicos. Rev. Gaucha Enferm. 2013; 34(2): 37-45.
18. Dainesi, SM. Pharmacovigilance: More than ever, an overall responsibility. Clinics. 2005; 60(4): 267 - 270.

19. Fontoura A. Utilização de medicamentos por gestantes em atendimento pré-natal em uma maternidade do município de Ribeirão Preto - SP [Dissertação]. Ribeirão Preto: Faculdade de Ciências Farmacêuticas de Ribeirão Preto, Universidade de São Paulo. 2009.

20. Silva RC, Santana AD, Santos AAP, Cerqueira GS. Plantas medicinais utilizadas na saúde da mulher: riscos na gravidez. Diálogos e Ciência. 2012; (32): 243-246. DOI: 10.7447/dc.2012.030.

21. Arcanjo GMG, Medeiros, MLFS, Azevedo RRS, Rocha TGM, Griz SAS, Mousinho KC. Estudo da utilização de plantas medicinais com finalidade abortiva. REB. 2013; 6(3): 234-250.

22. Nicoletti MA, Oliveira-Junior MA, Bertasso CC, Coporossi PY, Tavares APL. Principais interações no uso de medicamentos fitoterápicos. Infarma 2007; 19(1): 32-40.

23. Kesmodel U, Wisborg K, Olsen SF, Henriksen TB, Secher NJ. Moderate alcohol intake in pregnancy and the risk of spontaneous abortion. Alcohol Alcohol. 2002; 37(1): 87-92. DOI: 10.1093/ije/dyr189.

24. Souza GT, Rodrigues MC, Ciavaglia MC. Análise do grau de conhecimento da população sobre a teratogenia do álcool e a conduta de enfermagem. Rev. Bras. Enferm. 1996; 49(2): 287-304. DOI: 10.1590/S003471671996000200013.

25. Kroeff LR, Mengue SS, Schmidt MI, Duncan BB, Favaretto ALF, Nucci LB. Fatores associados ao fumo em gestantes avaliadas em cidades brasileiras. Rev. Saúde Pública. 2004; 38(2): 261-267. DOI: 10.1590/S003489102004000200016.

26. Moraes CL, Reichenheim ME. Rastreamento de uso de álcool por gestantes de serviços públicos de saúde do Rio de Janeiro. Rev. Saúde Pública. 2007; 41(5): 695-703. DOI: $10.1590 /$ S0034-89102007000500002.

27. ACOG. American College of Obstetricians and Gynecologists. Exercise During Pregnancy and the Postpartum Period No 267 [online]. Washington, 2002. Disponível em: http://www.acog.org. 